\title{
Early morning kidney transplantation: Perioperative complications
}

\author{
Mário Pereira Lourenço, Miguel Eliseu, Duarte Vieira Brito, João Carvalho, Edgar Tavares-Silva, \\ Lorenzo Marconi, Pedro Moreira, Pedro Nunes, Belmiro Parada, Carlos Bastos, Arnaldo Figueiredo \\ Urology and Renal Transplantation Department, Coimbra University Hospital Centre, Coimbra, Portugal.
}

\begin{abstract}
Summary Introduction: To reduce cold ischemia time (CIT), many kidney transplants are performed in the early morning. Conducting complex surgeries in the early morning may influence the surgeon's technical capacity and rate of surgical complications (SC). Aim: Evaluate the influence of surgery start hour (SSH) regarding duration of surgery (DS), immediate diuresis (ID), SC and acute rejection (AR); evaluate the influence of CIT regarding $S C, I D$, and AR. Methods: 2855 cadaveric transplants performed between June 1980 and March 2018 were retrospectively evaluated. Regarding SSH, two groups were created: Group M (00:00h-05.59h, $n=253)$ and Group D (06: 00h - 23: $59 \mathrm{~h}, \mathrm{n}=2602)$. Analyzing the impact of SSH on DS, ID, SC and AR. Evaluate the relationship between CIT (<18h, 18-30h and > 30h) on ID, SC and AR utilizing univariate and multivariate statistical analysis with SPSS. Results and Conclusion: Groups $M$ and $D$ were comparable in all evaluated demographic variables $(p>0.05)$, except cold ischemia time (Group M with higher CIT, $p<0.001$ ). Regarding univariate analysis, Surgery start hour did not influence DS $(p=0.344)$, and SC $(p=0.264)$, but related with higher ID $(p=0.028)$ and AR $(p=0.018)$. CIT related with immediate diuresis $(p=0.020)$ and acute rejection $(p<0.001)$ but did not relate with complications $(p=0.734)$. Regarding multivariate analysis, SSH only influenced immediate diuresis $(p=0.026)$ and did not influenced acute rejection $(p=0.055)$. CIT influenced immediate diuresis $(p=0.019)$ and acute rejection $(p<0.001)$. Surgery start hour influences Immediate diuresis. With this study, we conclude that the priority must be a short cold ischemia time.
\end{abstract}

KEY WORDS: Early morning transplantation; Kidney transplantation; Kidney transplant complications; Immediate diuresis; Surgery starting hour.

Submitted 9 January 2021; Accepted 23 February 2021

\section{INTRODUCTION}

Medical error and its influence on the health of patients is an important and current subject in modern medicine. In 1999, the Institute of Medicine of the United States of America reported that medical errors where responsible for one hundred thousand annual deaths and had a global cost of 29 billion dollars (1). Sleep deprivation increases the risk of medical error, so far as being called the Achilles heel of the medical profession (2). Sleep depri- vation can have serious consequences on the surgeon, affecting his ability to maintain his mental and motor abilities. Studies show that sleep deprivation can have the same effect as alcohol intoxication on the surgeon's abilities (3-6). It has been reported that there is an increase in the number of surgical complications and mortality when surgeries are performed after the professionals normal working hours (4-6).

Kidney transplant (KT), although not being an urgent procedure, is most of the times executed at late hours, even ate dawn. The reasons for such are several and include: the complex organization needed to distribute renal grafts, elective surgery that occupies surgical theatre and the need to prepare the receptor for surgery (7). The main reason for such high number of late procedures is the need to reduce cold ischemia time (CIT) as much as possible in KT, as larger CIT relates to worst graft function, smaller survival time of graft and higher patient mortality (8). Although, studies show that with an 18 hours window of CIT there isn't a significant difference in outcomes $(9,10)$. On the other hand, it has been described that surgical factors and the existence of complications influence the outcome of $\mathrm{KT}(11,12)$. As such, it is pertinent to address what can be more favourable, having the lowest CIT possible or having a "fresh" surgical team with hypothetically smaller number of complications. For reason external to the surgical team, CIT was longer in specific cases up to the point where it was relevant to decide between performing surgery at night or in the morning. If waiting until morning could cause CIT to be considered unsafe, procedures were conducted at later hours, being the group with the largest CIT the one that could not wait any more time. To our knowledge, there are only five large scale studies that studied this problem concerning Kidney Transplantation, with conflicting results and comparing different hours from the ones in this work $(7,13-16)$.

\section{Materials AND Methods}

Study design

Retrospective analyses of all KT occurring in the Urology and Kidney Transplant Service of the "Centro Hospitalar e Universitário de Coimbra”, Portugal, between June 1980 
and July 2018. Patients with combined transplant (pancreas, lung, heart and liver) and from living donor where excluded, being selected 2855 consecutive transplants.

The resulting population was divided into two groups based on the surgery start hour (SSH): Group M (00:00h05:59h) and Group D (06:00h-23:59h). Four outcomes where defined and measured based of SSH: duration of surgery (DS), the number of surgical complication (SC), immediate diuresis (ID) measured on the operating table and acute rejection (AR) of the graft.

The population was equally studied on the CIT and its influence in the ID, AR and DS.

Surgical team composition was not gathered in this study, but in the institution where the analysis was performed the presence of residents is not dependant of the hour of transplant. Being most teams composed of a senior surgeon and a resident.

Data collected was inserted in multiple labour laws, surgeons on the transplantation department are exclusively allocated to the task after normal working hours, having the right to rest after night procedures.

\section{Definitions}

Marginal donor was defined as having at least one of the following characteristics: over 60 years old, over 50 years and arterial hypertension, death by cerebral vascular accident or serum creatine level of over $1.5 \mathrm{mg} / \mathrm{dl}(17,18)$.

Acute rejection was defined as worsening of graft function on the first year, having been admitted a clinical diagnosis (increase in creatine blood level, imagological alteration, need for corticoid treatment) or histologic (kidney biopsy). Immediate diuresis was defined by the observation of urinary output in patients with no need for dialysis on the first week after kidney transplant. Surgical complications were divided in: urologic (urinary fistula, ureteric stenosis, ureteric necrosis), vascular (arterial or venous thrombosis, arterial stenosis, vascular anastomosis dehiscence), lymphocele, haemorrhage (need of surgical revision or transfusion), wound dehiscence, incisional hernia and others.

\section{Statistical analysis}

Baseline and demographic characteristics of group $\mathrm{M}$ and D were compared using the chi-squared test for categorical variables and the student's T test for continuous variables. Univariate analysis of the effects of SSH and CIT in outcomes were described using the chi-squared test. Multivariate analysis was conducted by applying the logistic regression method.

\section{Results}

\section{Demographic characteristics}

Between 00:00 hours and 05:59 hours there were 253 cadaveric transplants in contrast to 2602 in the other time period. Demographic characteristic of Group M and Group D are summarized in Table 1. In comparison, more patients from the group M presented CIT superior to 30 hours ( $<<0.001)$. All other characteristics were similar. Mean surgical time for group (group D is further divided) is summarized in Table 2 .
Table 1.

Demographic characteristic of the population.

\begin{tabular}{|c|c|c|c|}
\hline Populational demographic characteristic & Group M & Group D & $\mathrm{p}$ value \\
\hline Number of patients & 253 & 2602 & \\
\hline Marginal donor (\%/n) & $50.6 \%(128)$ & $50.2 \%(1307)$ & 0.932 \\
\hline Age of receptor (y) & $46.6 \pm 13.9$ & $45.6 \pm 13.7$ & 0.291 \\
\hline $\begin{array}{l}\text { Sex of receptor } \\
\text { Male }\end{array}$ & $69.2 \%(175)$ & $66.9 \%(1740)$ & 0.458 \\
\hline Right side implant & $79.8 \%$ (202) & $2131(81.9 \%)$ & 0.828 \\
\hline $\begin{array}{l}\text { Number of transplants }(\% / n) \\
\quad 1 \\
>1\end{array}$ & $\begin{array}{c}94.5 \%(239) \\
94.4 \%(2457)\end{array}$ & $\begin{array}{c}5.5 \%(14) \\
14.6 \%(155)\end{array}$ & 0.608 \\
\hline $\begin{array}{l}\text { Number of arteries of the graft } \\
1 \\
>1\end{array}$ & $\begin{array}{c}78.3 \%(198) \\
78.7 \%(2047)\end{array}$ & $\begin{array}{c}22.7 \%(55) \\
21.3 \%(553)\end{array}$ & 0.953 \\
\hline $\begin{array}{l}\text { Cold ischemia time }(\% / \mathrm{n}) \\
<18 \mathrm{~h} \\
10-30 \mathrm{~h} \\
>30 \mathrm{~h}\end{array}$ & $\begin{array}{c}38.3 \%(97) \\
53.9 \%(134) \\
8.7 \%(22)\end{array}$ & $\begin{array}{c}38.7 \%(1004) \\
58.6 \%(1521) \\
2.8 \%(72)\end{array}$ & $<0.001$ \\
\hline
\end{tabular}

Table 2.

Mean surgery duration time in relation to surgical starting time.

\begin{tabular}{|l|c|c|}
\hline Surgery start hour & $\%$ (n) & Mean duration of surgery (min) \\
\hline Grupo M & $8.8 \%(253)$ & $157.2(45-330)$ \\
00:00-05:59 & $12.9 \%(366)$ & $165.9(55-555)$ \\
\hline Grupo D & $149.1(45-615)$ \\
06:00-11:59 & $40.3 \%(1151)$ & $152.1(45-390)$ \\
$12: 00-17: 59$ & $38.0 \%(1085)$ & $153.5(45-615)$ \\
18.00-23:59 & 2855 & \\
\hline Total & & \\
\hline
\end{tabular}

\section{Univariate analysis}

The effects of surgical star hour in the outcomes defined (DS > 3 hours, ID, SC e AR) are summarized in Table 3. The effects of CIT on the same outcomes (except duration of surgery) are summarized in Table 4.

\section{Multivariate analysis}

The effects of SSH and CIT when analysed together (multivariate analysis) are summarized in Table 5.

Table 3.

Effect of surgery starting hour in outcomes of renal transplant.

\begin{tabular}{|l|c|c|c|}
\hline & Group M & Group D & p value \\
\hline Surgery duration > 3 hours & $20.6 \%(52)$ & $18.1 \%(472)$ & 0.344 \\
\hline Immediate diuresis & $71.1 \%(180)$ & $79.0 \%(2055)$ & 0.028 \\
\hline Surgery complications & $20.9 \%(53)$ & $18.1 \%(471)$ & 0.264 \\
Vascular & $5.9 \%(15)$ & $4.1 \%(107)$ & \\
Urologic & $5.5 \%(14)$ & $6.5 \%(168)$ & \\
Lymphocele & $2.0 \%(5)$ & $1.4 \%(37)$ & \\
Bleeding & $6.3 \%(16)$ & $3.3 \%(85)$ & \\
Wound dehiscence & $1.2 \%(3)$ & $1.6 \%(42)$ & \\
Abscess & - & $0.4 \%(11)$ & \\
Hernia & - & $0.4 \%(11)$ & \\
Other & - & $0.4 \%(10)$ & \\
\hline Acute rejection & $25.3 \%$ & $18.9 \%$ & 0.018 \\
\hline
\end{tabular}


Table 4.

Effects of cold ischemia time in the outcomes of kidney transplant.

\begin{tabular}{|l|c|c|c|c|}
\hline Cold ischemia time & $<\mathbf{1 8 h}$ & $\mathbf{1 8 - 3 0 h}$ & $>\mathbf{3 0 h}$ & $\mathbf{p}$ \\
\hline Immediate diuresis & $81.4 \%(900)$ & $77.2 \%(1277)$ & $66.0 \%(62)$ & 0.020 \\
\hline Surgery complications & $18.4 \%(203)$ & $18.1 \%(299)$ & $21.3 \%(20)$ & 0.734 \\
\hline Acute rejection & $14.4 \%(159)$ & $22.0 \%(364)$ & $41.5 \%(39)$ & $<0.001$ \\
\hline
\end{tabular}

Table 5.

Multivariate analysis of the effect of surgery starting hour and cold ischemia time in the outcomes of kidney transplant.

\begin{tabular}{|l|c|c|}
\hline Outcome & Variable & $\mathbf{p}$ \\
\hline Immediate diuresis & SSH & 0.026 \\
& CIT & 0.019 \\
\hline Surgery complications & SSH & 0.282 \\
& CIT & 0.796 \\
\hline Acute rejection & SSH & 0.055 \\
& CIT & $<0.001$ \\
\hline
\end{tabular}

\section{Discussion}

This work presents the highest number of patients analysed in relation to surgery start hour and its possible effects in kidney transplantation. Defined SSH was different when compared with other articles. In two different studies Kienz-Wagner et al. and Fechner et al. divided patients in groups of 08:00h-19:59h and 20:00h-07:59h $(7,14)$. Seow et al. divided SSH in 07:30h-17:59h; 18:00h-23:59h; 00:00h-07:29h (13), while Shaw et al. and Emmanouilidis divided the groups into periods of $3 \mathrm{~h}$ $(15,16)$. We chose to divide SSH in two blocks of 00:00h-05:59h and 06:00-23:59h such as to reinforce the effects of physical tiredness and sleep deprivation at dawn. Although, group M clearly presented a smaller number of patients, the high number of patients allows for reliable comparisons. Other distinct aspects are the outcomes studied, as most studies analyse the perioperative complications and renal graft survival. In this article we found favourable to try and relate fatigue of the surgeon and short-term outcomes, such as surgery duration, immediate diuresis and surgical complications. As short to median term outcome we evaluated the rate of AR.

The $\mathrm{M}$ and $\mathrm{D}$ groups are comparable in relation to all variables studied, except in CIT of the graft (Table 1), it being superior in group $M$. These results are logical, as they represent grafts that where implanted "at any hour" as a way to prevent achieving critical CIT time that could possibly stop the realization of the transplant.

Utilizing univariate analysis, SSH influenced significantly the ID and AR, not having influenced the DS or the number of SC (Table 3). In a similar significant analysis, CIT influenced the same outcomes (Table 4). As group $\mathrm{M}$ and $\mathrm{D}$ where distinct in relation to CIT, multivariate analysis was conducted (Table 5) showing that $\mathrm{SSH}$ influenced the ID but did not alter the rate of AR.

Kienzl-Wagner and Seow also did not find a relationship between SSH and the number of surgical complications while Fechner et al. demonstrated a larger number of vascular complications in KT conducted during the night period $(7,13,14)$. Shaw et al. in their analysis evidenced a larger number of urologic complications during 03:0005:59h and a higher number of any complications between 00:00-02:59h (15). Emmanouilidis et al. also demonstrated higher likelihood for the need of reoperation during the 03:00-05:59h interval (16).

Duration of surgery was not influenced by the SSH, although there isn't sufficient data from literature regarding this specific outcome. One Danish study, analysing the effects of sleep deprivation on the cognitive and technical abilities of surgeons, evidenced that surgeons can compensate for changes to their circadian rhythm, what may explain the results obtained in our study (19).

On this study, SSH significantly influenced ID. In the work by Kenzl-Wagner there was also a lower rate of ID in the nocturnal group (63.4\% vs. 69.9\%), although, without statistical significance. In that review, the author justified that tendency by the existence of a larger number of surgeries with marginal donor in the nocturnal group, what was not observed with our study. One simple justification could be that group $\mathrm{M}$ has a higher CIT, but changes to this outcome, ID, are maintain even after multivariate analysis. Notwithstanding the absence of a clear answer for this relation, it is known that a delay of graft function is associated with poorer outcomes and higher risk of graft loss, as show in a previous study done by our transplant centre (20).

The rate of AR in the $\mathrm{M}$ and $\mathrm{D}$ groups where $25.9 \%$ and $18.9 \%$, respectively. On multivariate analysis a distinct association between SSH and AR was not found, consistent with the results from Kienzl-Meyer et al. (14)

The key factor that influenced graft function was CIT. As it is showed by our work, CIT has a significant influence in ID and $\operatorname{AR}(8,10,21,22)$. Although, studies suggest similar outcomes in the CIT time frame of less than $18 \mathrm{~h}$, one study conducted by Debout et al. demonstrated that with every hour of CIT the probability of graft failure increases (hazard ratio: 1.013) and that the time frame of 18 hours can be suboptimal $(9,10)$. The same work stated the existence of a relation between CIT and graft failure (8).

Nevertheless, the recent study by Emmanouilidis et al., demonstrated that in the time frame of less than 23.5 hours of CTI, it is unfavourable to perform KT with the $\mathrm{SSH}$ in the 03:00-05:59h period (16).

The effects of sleep deprivation and of SSH has been studied in other specialities and surgeries. Regarding hepatic transplantation, Lonze et al. stated that surgery conducted in the nocturnal period was not associated with a higher number of complications but related with longer SD and risk of short term mortality (23). Analysing thoracic organ transplant, George et al. did not identify differences between outcomes in patients whose surgery was conducted at night (24). Other works in pancreatic surgery, heart, trauma surgery did not find worst outcome with late SSH (25-28).

There were some limitations to this study, starting with the retrospective analysis of collected data, the definition of early morning surgery (00:00-05:59) is arbitrary and may not relate precisely with the fatigue state of the surgical team. Regarding AR its characterization was expanded to include clinic and histologic diagnoses. It was decided not to assess the relation between SSH and graft survival 
as we considered there were no negative outcomes in the short term, and any data extrapolation would be too complex to explain.

\section{Conclusions}

Conducting KT at dawn showed no association with SD, $\mathrm{SC}$ or AR. However, it is connected with lower ID.

The CIT strongly influences ID and AR. In summary, data collected in this study support that SSH should prioritize CIT, making sure CIT is the lowest possible. This research did not receive any specific grant from funding agencies in the public, commercial, or not-for-profit sectors.

\section{AcKNoWledgment}

The authors of this article would like to give special reference to Doctors António Roseiro, Vitor Dias and Francisco Rolo whom without their continuous and valuable work in the area of Kidney transplant this work would not be possible.

\section{References}

1. Kohn L. To err is human: an interview with the Institute of Medicine's Linda Kohn. Jt Comm J Qual Improv. 2000; 26:227-34.

2. Gaba DM, Howard SK. Patient safety: fatigue among clinicians and the safety of patients. N Engl J Med. 2002; 347:1249-55.

3. Borman KR, Fuhrman GM; Association Program Directors in Surgery. "Resident Duty Hours: Enhancing Sleep, Supervision, and Safety": response of the Association of Program Directors in Surgery to the December 2008 Report of the Institute of Medicine. Surgery 2009; 146:420-7.

4. Chen CL, Chen Y Sen, Liu PP, et al. Living related donor liver transplantation. J Gastroenterol Hepatol. 1997; 12: S342-345.

5. Rothschild JM, Keohane CA, Rogers S, et al. Risks of complications by attending physicians after performing nighttime procedures. JAMA. 2009; 302:1565-72.

6. Gray A. United Kingdom national confidential enquiry into perioperative deaths. Minerva Anestesiol. 2000; 66:288-92.

7. Fechner G, Pezold C, Hauser S, et al. Kidney's nightshift, kidney's nightmare? Comparison of daylight and nighttime kidney transplantation: impact on complications and graft survival. Transplant Proc. 2008; 40:1341-4.

8. Debout A, Foucher Y, Trébern-Launay K, et al. Each additional hour of cold ischemia time significantly increases the risk of graft failure and mortality following renal transplantation. Kidney Int. 2015; 87:343-9.

9. van Roijen JH, Kirkels WJ, Zietse R, et al. Long-term graft survival after urological complications of 695 kidney transplantations. J Urol. 2001; 165:1884-7.

10. Opelz G, Döhler B. Multicenter analysis of kidney preservation. Transplantation. 2007; 83:247-53.

11. Król R, Ziaja J, Chudek J, Heitzman M, et al. Surgical treatment of urological complications after kidney transplantation. Transplant Proc. 2006;38:127-30.

12. Agüera Fernandez LG, Robles JE, Rosell D, et al. Análisis multivariado del impacto de las complicaciones quirúrgicas en el trasplante renal (Multivariate analysis of the impact of surgical complications in renal transplant). Arch Esp Urol. 1994; 47:999-1006.

13. Seow YY, Alkari B, Dyer P, Riad H. Cold ischemia time, sur- geon, time of day, and surgical complications. Transplantation. 2004; 77:1386-9.

14. Kienzl-Wagner K, Schneiderbauer S, Bösmüller C, et al. Nighttime procedures are not associated with adverse outcomes in kidney transplantation. Transpl Int. 2013; 26:879-85.

15. Shaw TM, Lonze BE, Feyssa EL, et al. Operative start times and complications after kidney transplantation. Clin Transplant. 2012; 26:E177-83.

16. Emmanouilidis N, Boeckler J, Ringe BP, et al. Risk balancing of cold ischemic time against night shift surgery possibly reduces rates of reoperation and perioperative graft loss. J Transplant. 2017; 2017:5362704.

17. Gopalakrishnan G, Gourabathini SP. Marginal kidney donor. Indian J Urol. 2007; 23:286-93.

18. Chapman JR. Marginal kidneys for transplantation. BMJ 2015; 351:h3856.

19. Amirian I. The impact of sleep deprivation on surgeons' performance during night shifts. Dan Med J. 2014; 61:B4912.

20. Moreira P, Sá H, Figueiredo A, Mota A. Delayed renal graft function: risk factors and impact on the outcome of transplantation. Transplant Proc. 2011; 43:100-5.

21. Salahudeen AK, Haider N, May W. Cold ischemia and the reduced long-term survival of cadaveric renal allografts. Kidney Int. 2004; 65:713-8.

22. Hernández D, Estupiñan S, Pérez G, et al. Impact of cold ischemia time on renal allograft outcome using kidneys from young donors. Transpl Int. 2008; 21:955-62

23. Lonze BE, Parsikia A, Feyssa EL, et al. Operative start times and complications after liver transplantation. Am J Transplant. 2010; 10:1842-9.

24. George TJ, Arnaoutakis GJ, Merlo CA, et al. Association of operative time of day with outcomes after thoracic organ transplant. JAMA. 2011; 305:2193-9.

25. Araujo RL, Karkar AM, Allen PJ, et al. Timing of elective surgery as a perioperative outcome variable: analysis of pancreaticoduodenectomy. HPB (Oxford). 2014; 16:250-62.

26. Heller JA, Kothari R, Lin HM, et al. Surgery start time does not impact outcome in elective cardiac surgery. J Cardiothorac Vasc Anesth. 2017; 31:32-36.

27. Zafar SN, Libuit L, Hashmi ZG, et al. The sleepy surgeon: does night-time surgery for trauma affect mortality outcomes? Am J Surg. 2015; 209:633-9.

28. Dalton MK, McDonald E, Bhatia P, et al. Outcomes of acute care surgical cases performed at night. Am J Surg. 2016; 212:831-836.

\section{Correspondence}

Mário Pereira Lourenço, MD (Corresponding Author) mariolourenco88@gmail.com

Miguel Eliseu - mgl.nobre@gmail.com

Duarte Vieira Brito - duartevbrito@hotmail.com

João Carvalho - joao.andre.mendes.carvalho@gmail.com

Edgar Tavares-Silva - edsilva.elv@gmail.com

Lorenzo Marconi - lorenzooliveiramarconi@gmail.com

Pedro Moreira - pedronetomoreira@gmail.com

Pedro Nunes - ptnunes@gmail.com

Belmiro Parada - parada.belmiro@gmail.com

Carlos Bastos - cabastos@netcabo.pt

Arnaldo Figueiredo - ajcfigueiredo@gmail.com

Urology and Renal Transplantation Department. Coimbra University

Hospital Centre Rua Maria Bourbon Bobone, n57, RE/esq, 3030-481,

Coimbra (Portugal) 